Cinémas

Revue d'études cinématographiques

Journal of Film Studies

\title{
Jean Renoir à Hollywood ou la recherche américaine d'une image française
}

\section{Roger Viry-Babel}

Volume 1, numéro 1-2, automne 1990

Américanité et cinéma

URI : https://id.erudit.org/iderudit/1000992ar

DOI : https://doi.org/10.7202/1000992ar

Aller au sommaire du numéro

Éditeur(s)

Cinémas

ISSN

1181-6945 (imprimé)

1705-6500 (numérique)

Découvrir la revue

Citer cet article

Viry-Babel, R. (1990). Jean Renoir à Hollywood ou la recherche américaine

d'une image française. Cinémas, 1(1-2), 72-85. https://doi.org/10.7202/1000992ar

\section{Résumé de l'article}

Dans l'étude de la réception critique des cinq films que Jean Renoir a réalisés aux États-Unis entre 1940 et 1946, l'auteur analyse comment la francité de Renoir s'est trouvée en contradiction avec un système de production américain. Il démontre que l'américanité des films a été mal reçue par le public français, parce que Jean Renoir inscrivait ses oeuvres dans une thématique française en utilisant le schématisme des films américains, ce qui ne pouvait que désorienter le public français. 


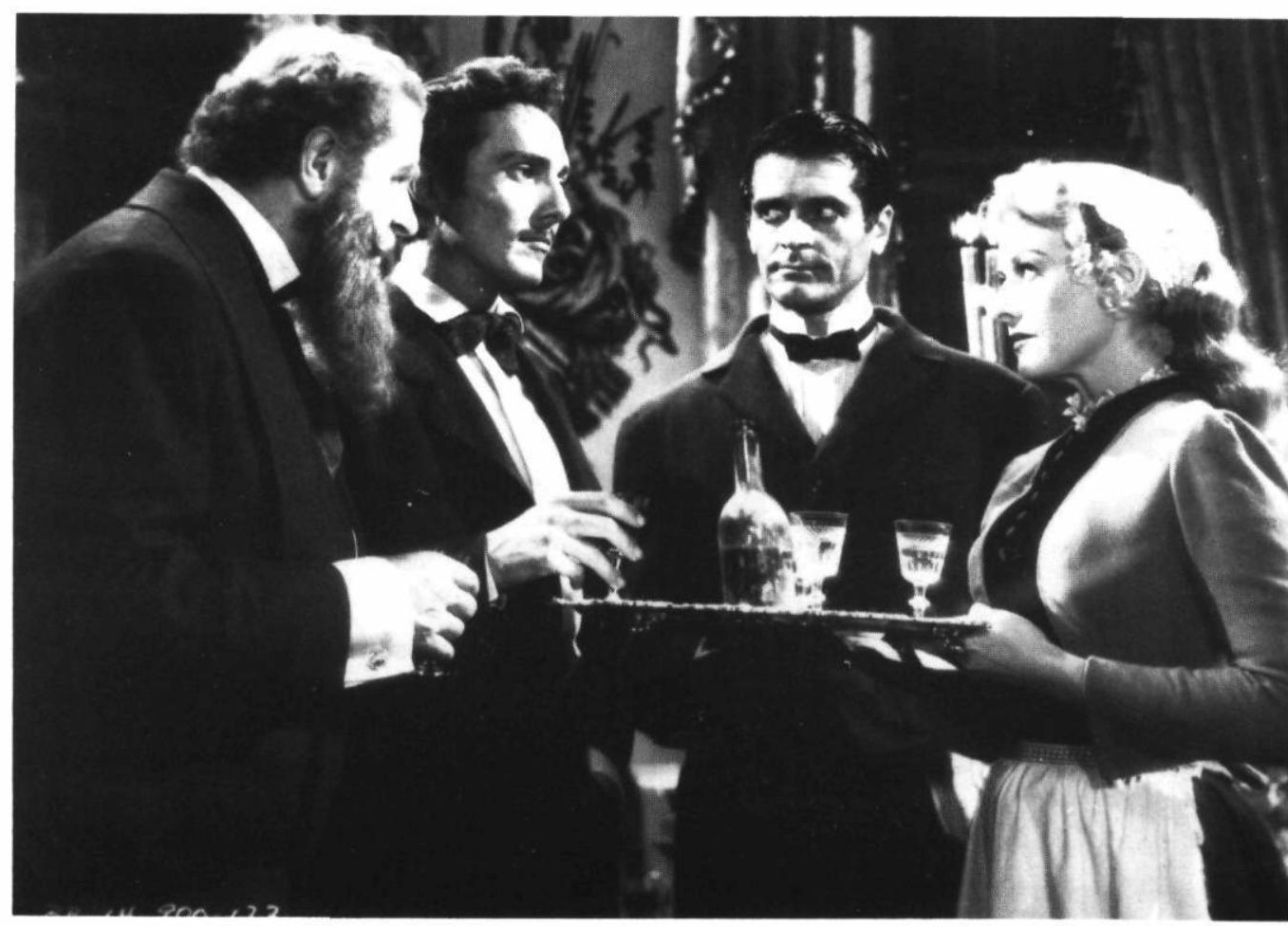

The Diary of a Chambermaid de Jean Renoir (1945) Coll. Cinémathèque québécoise 


\title{
Jean Renoir à Hollywood ou la recherche américaine d'une image française
}

Roger Viry-Babel

\section{RÉSUMÉ}

Dans l'étude de la réception critique des cinq films que Jean Renoir a réalisés aux États-Unis entre 1940 et 1946, l'auteur analyse comment la francité de Renoir s'est trouvée en contradiction avec un système de production américain. Il démontre que l'américanité des films a été mal reçue par le public français, parce que Jean Renoir inscrivait ses œuvres dans une thématique française en utilisant le schématisme des films américains, ce qui ne pouvait que désorienter le public français.

\begin{abstract}
In a study of the critical reception in the five films that Jean Renoir made in the United States between 1940 and 1946, the author analyses the conflict between the Frenchness of Renoir and the American film production system. He argues that the films were poorly received by audiences in France because Jean Renoir had created works with French themes but had used the schematism of American film, producing a combination which could only disorient the French public.
\end{abstract}

L'intitulé de ce texte peut paraître ambigu: Jean Renoir à Hollywood ou la recherche américaine d'une image française. S'agit-il d'analyser l'américanité de la représentation de la France dans les cinq films hollywoodiens de Renoir, ce qui présupposerait que Renoir, «metteur en scène français», s'est transmué en american director par le simple fait d'une transplantation, ou mieux s'agit-il 
d'analyser comment la francité de Renoir s'est trouvée en contradiction avec un système de production américain? J'entends bien ici par système de production, un appareil idéologique de production qui fait que la chaîne complète de production des studios hollywoodiens ne peut produire, quel que soit le réalisateur, le director, en un mot le maître d'œuvre, qu'une image américaine.

Le rôle de l'historien de cinéma, lorsqu'il a accès à la quasi-totalité des sources, est de recomposer patiemment ce fameux procès de production des films. Or malgré cet accès aux sources, je reste encore fort perplexe quant à la pertinence des remarques portées sur les films américains de Renoir. D'autant plus que l'ensemble des analyses produites çà et là sont souvent contradictoires: les 36 ouvrages généraux consacrés à Renoir, depuis la parution en 1953 de l'ouvrage de Calixto Cosulich à Rome, et 67 entretiens depuis 1945 offrent au chercheur une mosaïque d'opinions sans ligne directrice tangible. Renoir divise la critique et ne la rassemble que sur une seule certitude, celle d'être en face d'un auteur. Plus intéressante est l'opinion quasi unanime de la critique française entre 1945 et 1948 sur les films américains: «Mais qu'allait-il faire en Amérique?» Georges Sadoul écrit en 1948:
Le fromage de brie et les grisailles parisiennes manquent à ce grand homme si profondément français. On ne refait pas sa patrie en ma- quettes de studio. Vivre libre et Le Journal d'une femme de chambre nous l'ont prouvé. Depuis 1930 une règle de fer est établie à Holly- wood: confirmée par deux ou trois exceptions signées Hitchcock ou Fritz Lang, l'Amérique est désormais pour un Européen une cure de dévitaminisation, où son talent s'étiole et risque de périr ${ }^{1}$.

Rappelons rapidement les faits. Après l'offensive allemande de mai 1940, Renoir quitte rapidement le plateau de $L a$ Tosca, qu'il tourne en Italie. Il se réfugie aux Colettes à Cagnes. Robert Flaherty multiplie les démarches pour le faire venir aux États-Unis. Renoir hésite. «J'aurais honte à laisser tomber mes compatriotes quand tout va mal», lui écrit-il. Mais les pressions exercées afin qu'il accepte de travailler pour les nouveaux maîtres du cinéma européen l'incitent à partir. Après bien des difficultés, Renoir réussit à s'embarquer pour New York où il arrive le 31 décembre 1940. Le 15 janvier, il signe un contrat d'un an à la Twentieth Century Fox. Darryl F. Zanuck (un producteur qui va jusqu'à choisir les cravates de ses interprètes) aimerait lui imposer un sujet français ou, à la limite, européen. Renoir, au contraire, tient à un sujet américain. Le premier film sera Swamp Water à partir d'un scénario de Dudley Nichols, tiré d'un roman populaire de Vereen Bell paru en feuilleton l'année précédente dans le Saturday Evening. Renoir écrit: 
Swamp Water, ce qui signifie en français marécage, est le premier film que j'ai tourné en Amérique. J'ai choisi ce sujet pour deux principales raisons: la première est que le scénario est l'œuvre d'un écrivain pour qui j'ai une grande admiration, Dudley Nichols. La deuxième, c'est parce que cette histoire est typiquement américaine, sans aucune influence étrangère. Ma destinée m'ayant amené à devoir faire des films en Amérique, j'ai pensé que la seule façon de les faire proprement, c'était d'entrer résolument en contact avec la nature et les hommes de ce pays. Swamp Water a été mon introduction à l'Amérique.

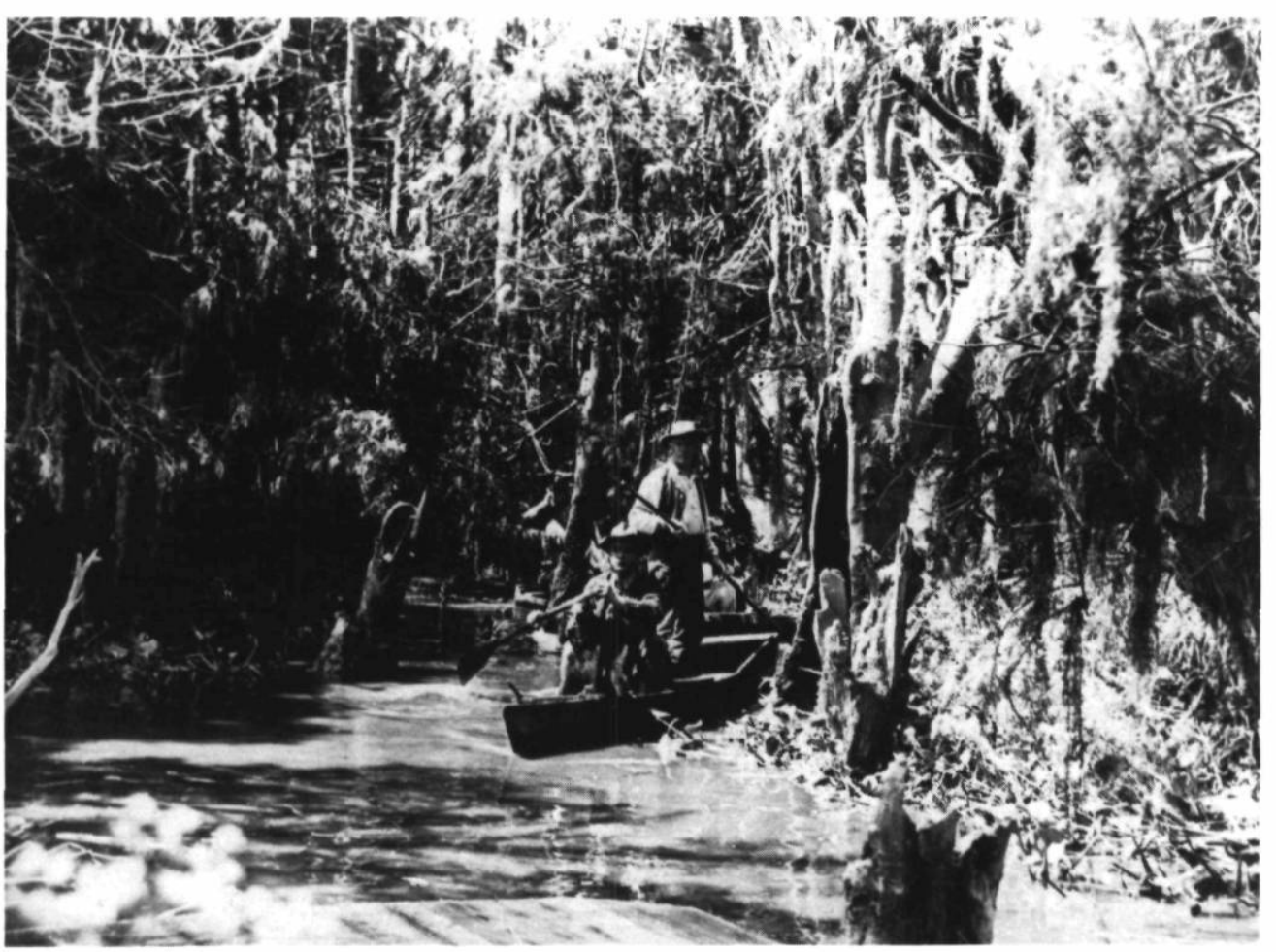

Swamp Water de Jean Renoir (1941)

Coll. Cinémathèque québécoise

En 1942, Renoir signe un contrat avec la RKO après avoir travaillé plusieurs semaines pour Universal sur Amazing Miss Hollyday, une comédie musicale sirupeuse écrite pour Deanna Durbin que signera finalement son assistant Bruce Manning: 
Pour être tout à fait franc, je dois t'avouer que quelle que soit la gentillesse de Deanna Durbin ou des autres, je commençais à en avoir assez de ce bain de sirop (...). Je suis exactement dans l'état de l'amant de la femme du pacha turc amateur de parfum qui avait dû se cacher dans un placard rempli de flacons odoriférants et qui en sortant hurlait en défaillant: de la merde!, par pitié de la merde! (écrit dans une lettre à son fils en date du 3 août 1942).

Le premier film pour RKO est This Land is Mine dont l'accueil public est très chaleureux. Charles Laughton et Dudley Nichols sont leurs témoins. En mars-avril 1944, pour l'Office of War Information, Renoir et Garson Kanin tournent un court métrage, Salute to France, destiné à présenter la réalité française aux G. I. qui vont débarquer. De septembre à novembre 1944, Renoir tourne The Southerner, dont la sortie en Amérique en avril 1945 provoquera des remous dans l'opinion publique. Le Sud ne supporte pas qu'un Européen adapte une histoire d'ouvriers au Texas.

«Quand on a assez peu de sens pratique, en pleine guerre, pour risquer de l'argent dans un film pareil, au moins, pour l'amour du ciel, qu'on s'adresse à un homme du Sud! Autant que possible, un homme du Sud avec un peu plus de nerfs et de réactions devant la vie qu'un romancier», écrit ironiquement un scénariste d'Hollywood qui explique pourquoi le maire de Memphis a interdit le film dans sa ville. Ce maire a décrété l'interdiction parce que «ce film donne l'impression que, dans le Sud, les Blancs sont une racaille ignorante (...). Ce film a l'air de dire qu'on vit très mal dans le Sud. Les ouvriers agricoles y sont représentés sous un jour très désagréable». La polémique servit en fait au succès d'exploitation du film, qui fut finalement couronné par la Palme du meilleur acteur et du meilleur réalisateur, décernée par la critique new-yorkaise en 1945 avant de recevoir le Lion d'or à la Mostra de Venise.

De juillet à septembre 1945, Jean Renoir dirige The Diary of a Chambermaid, production indépendante de l'acteur Burgess Meredith qui en est également le scénariste et l'interprète principal aux côtés de sa femme Paulette Goddard. Renoir réalise ici un de ses rêves de jeunesse: porter à l'écran le roman d'Octave Mirbeau dont il s'était déjà inspiré pour l'écriture de son premier film, Catherine ou Une vie sans joie. La critique est bonne et le film figure en huitième position des 10 meilleurs films de l'année en Angleterre.

En février 1946, Renoir tourne Woman on the Beach avec bien des difficultés. Il est prêt plusieurs fois à abandonner le projet. Il doit, à la suite d'une avant-première désastreuse, retourner plus du tiers du film et modifier le montage. La nouvelle mouture se 
conclura sur un désastre financier. Renoir ne tournera plus à Hollywood, même si The River est une production américaine. Tous ces films seront distribués dans le désordre, à Paris, entre juillet 1946 et mai 1950 dans des versions françaises déplorables.

Si nous mettons a part Salute to France, mélange habile de fiction et de documentaire, qui est un court métrage de propagande, seuls deux films, This Land is Mine et The Diary of a Chambermaid, se situent en France. Encore faut-il préciser que This Land is Mine, abusivement traduit par Vivre libre, n'est pas sensé se dérouler en France. Le carton explicatif du début du film le précise: "Somewhere in Europe. This town is half the World today: we fight to keep it from being all the World tomorrow."

Le script est lui-même fort explicite:

1/Ouverture Gros plan sur un drapeau qu'on abaisse sur un fond de ciel sombre: We cannot see the nationality of the flag.

2/Exterior french street-day

3/Exterior french street-another section

4/Exterior-New York street

5/Exterior-Cimarron street

et ainsi de suite, une succession de plans opposant des rues de New York et des rues françaises envahies par les soldats allemands.

Une note en début du script précise: «Le lieu est symbolique de tous les pays occupés et aucun n'est précisément identifiable. Aucun des décors ou des comportements ne doit faire trop "étranger". Nous réalisons une transposition totale d'un pays étranger mais on y parlera anglais sans aucun accent à l'exception des Allemands. Les personnages parlent comme vous le faites, ils ont les mêmes habitudes que les vôtres, ce pourrait être vous, tout sera traduit en anglais, y compris les pancartes dans les rues et les titres des journaux.»

La volonté d'universalisme de Renoir et de Nichols sera parfaitement saisie par le public américain. This Land is Mine, littéralement: Ce pays est le mien, appartient bien à cette longue série de films interventionnistes destinés à persuader l'Américain moyen du bien-fondé de l'intervention américaine aux côtés de l'Angleterre contre le régime nazi.

Malheureusement, deux phénomènes indépendants l'un de l'autre vont rétablir le caractère français de la production et provoquer l'irritation du public hexagonal lorsqu'il le découvrira en juillet 1946. Tout d'abord, la version française est non seulement traduite mais «réadaptée»sans demander l'avis du réalisateur. On filme des gros plans traduits des inscriptions en anglais. Le socle de la statue 
du monument aux morts de la Grande Guerre se francise, les affiches en anglais des occupants allemands deviennent d'authentiques affiches telles qu'ont pu les voir les Français de zone occupée, les journaux retrouvent eux aussi leur caractère gaulois. Mieux, des deux phrases préliminaires du film, la traduction française ne garde que le «quelque part en Europe» et élimine l'universalisme de la seconde partie. Rien d'étonnant dès lors que l'assimilation à une situation française ne provoque l'irritation des critiques et du public:

Sans doute, n'aurait-il pas fallu consacrer plus de dix lignes à ce film s'il ne portait la signature de Jean RENOIR, le plus grand réalisateur français. Qu'on nous permette de tenir cette signature pour un faux en écriture. Non pas que RENOIR n'ait jamais fait de mauvais films, mais parce que ce Français buveur de vin rouge, l'eût-il voulu, n'aurait su peindre son pays avec les couleurs du lait froid, et le chanter avec le lyrisme d'un cantique presbytérien du Minnesota ${ }^{2}$.

Ce que l'on ne comprend plus du tout, c'est que les auditeurs de ces films de circonstances n'aient pas résolument passé par pertes et profits ce travail de guerre qui n'offre plus qu'un intérêt documentaire, et prétendent infliger AUJOURD'HUI aux Français une désobligeante caricature payante du drame même dont ces Français ont été les vrais acteurs, les vrais héros, les vrais traîtres, les vraies victimes (...). Il y a là au moins un manque de tact qui justifie l'irritation de la critique et du public ${ }^{3}$.

On peut multiplier à l'infini les citations. Toutes se résument en fait au titre d'une critique d'André Bazin dans L'Écran français du 17 juillet 1946: «La résistance française à l'usage des Chinois.» En fait, cette incompréhension est aggravée par un phénomène qui relève à mon sens parfaitement de la notion d'américanité. Ce second phénomène est lui aussi quasiment indépendant de la volonté de Renoir. Peut-être d'ailleurs s'explique-t-il par le caractère influençable du réalisateur. Ce démocrate sincère, compagnon de route du parti communiste pendant le Front populaire, n'était-il pas «tombé amoureux» successivement de Louis XVI pendant le tournage de La Marseillaise, et du fils de Mussolini lors d'une tournée dans les universités italiennes au début de 1940, au point de faire basculer idéologiquement ce qui devait être le grand film de la gauche française pour le Cent cinquantenaire de la révolution, d'accepter de se compromettre avec les fascistes italiens ou encore de faire des déclarations douteuses à la presse fasciste de Salazar lors de son transit par Lisbonne sur la route de la libre Amérique?

Dudley Nichols, le scénariste de This Land is Mine et de Swamp Water, représente aux yeux de Renoir non seulement le grand cinéma hollywoodien de qualité mais encore un scénariste «engagé» à 
qui l'on doit Stagecoach (1939), The Informer (1935), The Long Voyage Home (1940). Par lui, Renoir se rattache à la grande tradition fordienne et à une inscription sociale affirmée dans tous les films de la même époque (Grapes of Wrath, 1940; Tobacco Road, 1941; ou How Green Was My Valley, 1941). Renoir, pour d'autres raisons qui tiennent à sa passion des acteurs, est également en admiration devant Charles Laughton, par ailleurs fin amateur d'art, qui possède une version admirable du Jugement de Paris d'Auguste Renoir.

Cela ne signifie pas pour autant que Jean Renoir ne maîtrise pas le scénario ou qu'il ne dirige pas l'acteur. Mais convaincu qu'il ne fait pas un film français, qu'il vise l'universel à travers une histoire malgré tout française - le scénario ne s'inspire-t-il pas librement de la nouvelle d'Alphonse Daudet, La Dernière Classe, qui illustre l'attachement d'un instituteur alsacien pour la France lors de l'occupation prussienne de 1870 ? - Renoir réalise un film schématique, conforme à la tradition américaine.

Un des défauts mais aussi des qualités du cinéma hollywoodien de cette époque n'est-il pas la fonction emblématique des personnages? Von Keller, le major allemand, est l'incarnation de toutes les déviances nazies:
Vous voyez je suis franc, je n'ai rien à cacher. Je vous dis ces choses parce que vous êtes un homme d'intelligence. Lambert n'était qu'un outil, très honnête mais pas très brillant. Le maire - et vous aviez raison à l'audience - ne travaille que dans son propre intérêt mais nous avons besoin d'eux et nous en trouvons dans chaque pays que nous envahissons (sourire). Mais même en Allemagne, nous les utilisons. C'est ainsi que notre parti est arrivé au pouvoir. Il y en a partout, c'est pourquoi rien ne peut nous arrêter dans notre conquête du monde. L'Amérique se sent en sécurité à cause des océans - ils pensent en terme d'armée et d'avions - mais ils sont déjà envahis. Les honnêtes Lambert et les malhonnêtes Manville nous attendent pour nous accueillir juste comme ils l'ont fait en Europe. Et si à n'importe quel moment nous avons besoin de faire la paix - à condition que la paix soit un moyen de conquête - leur patriotisme sincère trouvera autant d'arguments qu'il le faut pour la paix (rires). Après tout, qu'est-ce que les États-Unis? Un charmant cocktail d'Irlandais et de Juifs. Très spectaculaire mais un peu infantile. Et l'Angleterre, quelques vieilles "ladies" portant les pantalons de leurs grand-pères (...). Lory, je suis ravi que vous ayez décidé de vivre et d'être un homme libre. Vous êtes instituteur et vous avez une grande tâche, la régénération de la jeunesse. Vous avez à la préparer au monde de demain et croyez-moi, ce sera un monde parfait ${ }^{4}$.

Nichols et Renoir prennent soin à partir d'une situation historique essentiellement française de dépasser cette réalité pour atteindre à un discours moral sur la compromission, destiné quasi exclusivement aux Américains, qui représentent le dernier bastion de la liberté. Mais au-delà du regard moral qui repose sur un dis- 
cours idéologique sans faille, se profile également toute une série de clichés sur l'Européen et plus particulièrement sur le Français. On peut en souligner quelques-uns: la présentation des jeunes filles, mélange de légèreté et de volonté en même temps qu'objet de désir, le souci de la nourriture compris non seulement comme une préoccupation essentielle de l'époque (marché noir, raréfaction, etc.) mais comme signe distinctif d'une culture (le lait, le pain, etc.), le caractère des costumes avec l'opposition entre la modernité du costume de l'institutrice et l'apparence obsolète de celui d'Albert Lory. Tous ces signes sur lesquels se greffent des comportements caricaturaux (obséquiosité dans les rapports de politesse qui tranche avec l'aspect direct des rapports américains) augmentent l'irritation de la critique française d'autant plus que Renoir, dans la peinture des rapports de force entre les personnages de ses films français précédents, avait «gommé» tout schématisme comportemental et accordé à chacun d'eux un intérêt égal dans le refus du manichéisme. L'art de Renoir, dans la représentation sociale, était bien d'offrir toutes les chances à tous les personnages, même s'il était convaincu que le drame du monde «tient à ce que chacun a ses raisons». Les points de vue de deux critiques sont ici éloquents de la réception française:

C'est la résistance intérieure qu'on nous dépeint. Nous y voyons une France (ou tout autre pays) curieuse, où toutes les habitudes sont anglo-saxonnes, ainsi que le décor de la rue et des intérieurs. Nous y voyons des Allemands de mélodrame et des résistants bavards, qui font en Cour d'assises (qu'on appelle curieusement Cour de justice) de longs discours sur la nécessité de combattre l'occupant, malgré la présence d'officiers allemands qui encaissent sans mot dire. (...) Malheureusement on ne sait pas de quoi on parle, ce qui est excusable quand on est sur la côte de la Californie. Ce qui n'est pas excusable par contre, c'est, ignorant tout de la France occupée, de vouloir la décrire. Si même l'on admet la nécessité à l'époque de faire de tels films de propagande, on ne comprend pas que des Français aient pu à ce point méconnaître l'atmosphère et le détail de la vie française, on ne comprend pas surtout qu'on ose présenter ces films au public français 5 .

On n'aimerait pas traiter M. Jean Renoir comme un récidiviste du navet et l'auteur de La Grande Illusion a quelques excuses, on l'imagine, pour avoir tourné en Amérique un film stupide et offensant. Il est arrivé en émigré, dans un pays qui ne le connaissait guère et il lui a fallu passer par quelques exigences hollywoodiennes. Mais je tiens qu'un véritable artiste n'a jamais à être excusé. Le sculpteur brise sa statue manquée, le peintre brûle sa toile où il a bredouillé. Il forge sa propre liberté. Celui qui présente au public une œuvre signée de son nom en porte toute la responsabilité. En l'occurrence celle de Jean Renoir est très lourde ${ }^{6}$. 
Mais le même reproche ne sera-t-il pas fait à Fritz Lang pour son film The Hangmen, Also Die, écrit d'après un scénario de Brecht et dont la reconnaissance des qualités prendra, ici encore, plusieurs décennies?

Avec The Diary of a Chambermaid, le problème est encore plus aigu. Renoir joue clairement la carte française. Finalement, il répond à ce que Zanuck exigeait de lui: faire des films typiquement français à Hollywood. Ici encore la critique française sera impitoyable.

Mais il faut préciser que si l'accueil de la critique américaine avait été bon, le public américain n'avait guère prisé le mélange des genres contenu dans le film. Dès la sortie même, certains critiques américains avaient émis des doutes:

Lorsque la foule en colère tue le méchant valet sur la place du village on peut douter que ceci constitue un «happy ending». Tout comme on peut douter que le public américain partagera beaucoup de ce que le réalisateur français Jean Renoir offre en guise de comédie. À la première, il y avait des rires et des faux applaudissements à contretemps, tandis que des silences de mort accompagnaient des séquences qui auraient dû amuser. Meredith endosse la seule responsabilité d'un scénario tiré d'un roman et d'une pièce étrangère. Son interprétation bondissante du vieil original ne vient peut-être pas de lui mais en tant que coproducteur, il l'accrédite. Est-ce que quelqu'un a visionné quotidiennement les rushes? Il est impensable que Bogeaus ou Meredith ne se soient pas rendu compte que ces touches de "social significance» du script sont mal placées et par conséquence sans portée. United Artists peut commercialiser Diary of a Chambermaid sous son titre incitateur et sur la qualité des participants. On ne doit pourtant pas s'attendre à une longue carrière et des critiques positives ou contradictoires ne seront d'aucun aide?

Mais revenons à l'accueil francophone:

Du Jean Renoir à la sauce hollywoodienne. Recette étrange aux résultats déconcertants ${ }^{8}$.

On a peine à croire que c'est vraiment Jean Renoir, le Jean Renoir de La Bête humaine et de La Grande Illusion qui a mis en scène cette production où le ridicule voisine avec le grotesque et le fabriqué9.

Une suite manquée à La Règle du jeu ${ }^{10}$.

Le film est tiré avec une désarmante pudeur du roman de Mirbeau. Dire que Mirbeau a été trahi serait un euphémisme: Burgess Meredith, le scénariste, a escamoté Mirbeau. Cette fois-ci, le naturalisme est bien mort»11. 
Ce qui plaisait à Renoir dans le roman de Mirbeau, c'était le style d'une époque qui fut celle de la peinture impressionniste et une violence qui, à la manière de Pot-Bouille, décrit une société par son linge sale et ses eaux polluées. La censure américaine a châtré cette vigueur et, d'autre part, l'inculture d'Hollywood touchant à la couture, la construction des décors ou les éclairages a presque réduit à néant les tentatives faites par le fils d'Auguste Renoir pour retrouver l'air du temps que respirent les personnages du Moulin de la galette ${ }^{12}$.

Renoir s'est fait doublement piéger. À la fois par son sujet et par les conditions de tournage. Vouloir adapter à l'écran, en 1945, Le Journal d'une femme de chambre d'Octave Mirbeau, c'est s'exposer à faire des choix cruciaux qui lui seront de toute façon reprochés, quelles que soient la réussite et la qualité du produit final.

Le premier de ces choix concerne le «naturalisme» de l'œuvre. La remarque de Georges Sadoul est parfaitement légitime. Mais son «antiaméricanisme» mérite d'être nuancé: ce n'est pas seulement aux États-Unis que la censure ne tolérait pas que l'on reproduisît avec toute la fidélité réaliste les comportements des personnages du Journal! Nul doute que nous n'ayons eu en France, à la même époque, de fervents adeptes du code Hayes qui auraient émasculé le film avec allégresse s'il s'était permis quelque volonté d'être scrupuleusement fidèle à l'original. Il fallait donc, pour Renoir, tirer le scénario vers la comédie sociale, plutôt que vers la comédie de mœurs.

Le fait de réaliser ce film au moyen d'une production indépendante est, de plus, un double leurre. Tout d'abord, parce que le système de distribution en aval du tournage est là pour faire respecter certaines normes commerciales qui sont en fait des normes morales; ensuite, parce que l'un des producteurs n'est autre que le scénariste et l'acteur principal du film, et en plus le mari de la vedette. Malgré les qualités évidentes de comédien de Burgess Meredith, il ne sera jamais Chaplin!

Il est évident, et Renoir me l'avoua en 1976, qu'il fit autant le film par passion pour une histoire qu'il avait déjà évoquée dans son premier film, que pour le plaisir de diriger Paulette Goddard. Pour lui, c'était avant tout l'héroïne des films de Chaplin, celle à qui il avait inconsciemment rendu hommage dans la dernière séquence des Bas-fonds, avec cette citation non déguisée des Temps modernes...

Il arrive à ce film la même mésaventure - mais ne serait-ce pas plutôt une aventure heureuse? - que celle relative au tournage de La Marseillaise. Au cours du tournage, par passion pour ses per- 
sonnages, et leurs interprètes, Renoir infléchit lentement le sens du film. Il en est conscient, et porte toute son attention sur la transposition non plus d'une époque - quel costumier, quel décorateur travaillant à Hollywood dans les années 40 peut réussir à conférer une once d'authenticité à un décor français? - , mais des retrouvailles avec ce qu'il appelle «le geste français». Et c'est ce qui, à mon sens, fait la qualité du film aujourd'hui. Paul Gilson affirmait à ce sujet:

\begin{abstract}
Parmi les fleurs de son jardin d'Hollywood, Jean RENOIR a reçu la visite de fantômes de France: ceux du cantonnier, du limonadier, du facteur, du chef de fanfare et de l'employé du Chemin de fer auquel il prête les couleurs des tableaux de son père. Avec lui, qu'il s'agisse d'un soufflet, d'une pompe à eau dans une cour de ferme, ou d'un bougeoir posé sur une chaise de paille, les objets usuels deviennent aussitôt des objets poétiques. À voir le Journal d'une femme de chambre on se demande si Jean RENOIR n'a pas retrouvé le bock oublié sur la table d'Édouard Manet et le canotier de SEURAT, perdu dans l'Île de la Grande Jatte. (...) Que ce soit Paulette GODDARD (Célestine), Judith ANDERSON (Mme Lanlaire), Hurd HATFIELD (Georges), Francis LEDERER (Joseph), Reginald OWEN (Mr Lanlaire), les acteurs jouent dans les décors du Mesnil avec une telle vérité qu'ils paraissent interpréter la version américaine d'un film français. Mais il convient de signaler spécialement Burgess MEREDITH car, pas plus que celui de Charlot, nous ne pourrons oublier désormais le personnage extravagant qu'il a su créer: ce Capitaine Mauger qui croque les roses en semaine et garde les lys d'eau pour son dessert du dimanche. À la fin du film, ce n'est plus qu'un pantin cassé dont la montre oscille au bout d'une chaîne d'or et qui mime une dernière gigue sur l'épaule de son meurtrier ${ }^{13}$.
\end{abstract}

Tout ceci est du Renoir de la plus pure eau. En travaillant en studio, tout en sachant qu'il n'atteindra pas à un quelconque réalisme par la recherche du détail vrai, il détourne son propos en réalisant une suite de tableaux, ou plutôt de poses picturales directement inspirées par les tableaux paternels. Faisons toutefois remarquer que le texte de Paul Gilson est une des rares critiques positives de l'époque.

Quarante-cinq années ont passé depuis. Les films américains de Renoir prennent leur place dans une œuvre qui couvre près d'un demi-siècle de cinéma et dont la cohérence n'avait guère, alors, été mise en valeur. J'emploie volontairement la dénomination de films américains, même s'ils s'inscrivent dans une thématique française. Leur américanité a été mal reçue par le public français, dans la mesure où, tout d'abord, Jean Renoir incarnait toutes les vertus françaises au plus haut niveau. Mais surtout, parce que, dans sa volonté de schématisme, le cinéma américain ne pouvait que désorienter le public français.

Pour ne prendre qu'un exemple, le début du Diary of a Chambermaid multiplie les accessoires d'époque (costume de Célestine, 
carton à chapeau, etc.) pour historiciser son propos. La musique qui accompagne vient alors comme «sursigne» de la francité. Il s'agit d'une série de variations insistantes sur l'air de la chanson Fascination, scie musicale célèbre du début du siècle qui insiste sur une image caricaturale de la femme. Ce fait se voit accentué lors de la séquence sans paroles de la présentation des activités de Célestine: suite de plans enchaînés de la femme de chambre, tirant l'eau à la pompe, faisant chauffer l'eau de la lessive, essorant le linge, lavant le carrelage à grande eau. Toutes ces activités, qui semblent inspirées de croquis d'Auguste Renoir, sont soulignées par un pot-pourri d'airs de chansons populaires, voire de comptines enfantines françaises. Ce procédé fait partie de l'arsenal narratif américain. Les spectateurs de l'époque n'y voient aucun effet de redondance. Il s'agit plutôt pour eux de ce que j'appelle un «sursigne» ou effet d'insistance. Le procédé ne s'applique pas qu'aux films dont l'action présente un caractère exogène pour ne pas dire exotique. La citation musicale du Chant de départ dans le générique de début et de fin de This Land is Mine, la citation de $\mathrm{La}$ Marseillaise dans Casablanca de Michael Curtiz s'apparentent aux citations de chants traditionnels du Sud dans Swamp Water. Ce procédé fonctionne toujours, même si les compositeurs et les réalisateurs y introduisent aujourd'hui une distanciation ironique. Il fait toujours sourire. S'il est admissible lorsqu'il se trouve utilisé par Ford, Hawks ou Huston, il apparaît comme une insupportable concession à l'américanisme lorsqu'il se retrouve dans un film de Clair, de Duvivier ou de Renoir.

Ce que l'américanisme - j'entends par ce terme l'ensemble des manifestations d'américanité décelables dans un film — n'a pas su faire disparaître dans les films de Renoir, c'est la volonté cosmogonique qui s'y révèle. Qu'il tourne sur les bords du Loing l'histoire de La Fille de l'eau, qu'il nous entraîne au bord des marais d'Okefenokee en compagnie de la sauvageonne de Swamp Water, ou qu'il nous raconte les premiers émois d'une jeune Anglaise sur les rives du Gange dans The River, c'est toujours la même façon de montrer ce moment précieux et rare qui accompagne chez toute jeune fille et dans toute civilisation la naissance à l'amour. Que le château des Lanlaire soit reconstruit à côté de Sunset Boulevard par Eugène Lourié, qui six ans plus tôt avait reconstruit le château Solognot de La Règle du jeu sur les bords de La Seine, que l'Irlandaise Maureen O'Hara incarne à Hollywood une jeune institutrice française ne changent rien à l'affaire. Les oppositions de classes, les divisions verticales, l'esprit de caste et les idéologies étriquées prennent aussi bien racine sur les bords de la rivière San Joaquim dans The Southerner, que sur les bords de l'étang de Berre dans Toni. «On ne conte jamais que la même histoire, on ne 
creuse jamais que dans le même trou» confiait Auguste Renoir à son fils. Jean Renoir, en me rapportant ces propos, ajoutait: «J'ai l'impression d'avoir creusé dans plusieurs trous, mais toujours dans la même direction.»

L'histoire a relativisé les jugements des contemporains de Jean Renoir. Ses films américains sont réintégrés à leur juste place dans la lente, patiente et parfois malhabile construction d'un univers sans que jamais l'architecte Renoir n'ait eu à l'esprit un plan directeur ou un schéma général. Pour lui, un geste, un regard d'actrice peuvent valoir davantage qu'une grande tirade ou qu'un impeccable mouvement de grue.

Université de Nancy II

\section{NOTES*}

1 Les Lettres françaises (13 mai 1948).

2 Georges Sadoul, Les Lettres françaises (1947).

3 André Lang, France-Soir (1946).

4 Traduction littérale du texte original.

5 Raymond Jarry, Journal du Dimanche (28 juillet 1946).

6 Jean Fayard, Opéra (24 juillet 1946).

7 Traduction de propos recueillis dans le Los Angeles Times (28 janvier 1948).

8 Le Soir (Bruxelles, 27 février 1940).

9 Le Phare (27 février 1948).

10 Les Lettres françaises (15 juin 1948).

11 Jacques de Baroncelli, Le Monde (19 juin 1940).

12 Georges Sadoul, op. cit. (1 $1^{\mathrm{er}}$ juillet 1948).

13 Paul Gilson, L'Écran français (20 mars 1946).

* Étant donné l'utilisation exceptionnelle de sources historiques rares et, pour ces raisons, incomplètes selon le modèle de références adopté par la revue, la direction a choisi de reporter toutes les informations en notes et de ne pas tenir compte de la section «ouvrages cités». 